\title{
Desarrollo regional e innovación social en el autoempleo: estudio del caso de los programas de reactivación comarcal como iniciativa pública ${ }^{1}$
}

\author{
Garazi Azanza Martínez de $\operatorname{Luco}^{2}\left(\mathbb{0}\right.$, Anaïs Iglesias Sánchez $^{3}{ }^{\complement}$, Garbiñe Henry Moreno ${ }^{4}$ y Beatriz \\ Alejos Garmendia ${ }^{5}$ (D)
}

Recibido: 22 de julio de 2019 / Aceptado: 5 de noviembre de 2019 / Publicado: 19 de mayo de 2020

Resumen. La aplicación de la innovación social en el ámbito de la empleabilidad es cada vez más reconocida por las instituciones. Desde la Diputación Foral de Bizkaia se ha implementado el Programa de Reactivación Comarcal (PRC) para la promoción del autoempleo, principalmente entre personas jóvenes, personas desempleadas de larga duración y mayores de 45 años con especiales dificultades de reincorporación al mercado de trabajo. El objetivo de este artículo es analizar el PRC como buena práctica de innovación social en materia de empleo.

Palabras clave: Empleo; Autoempleo; Innovación social; Desarrollo regional; Políticas públicas; Empoderamiento.

Claves Econlit: E24; J08; L26; L38; O35; R58.

[en] Regional development and social innovation in self-employment: case study of local reactivation programs as a public initiative

Abstract. The practice of Social Innovation in the field of employment is increasingly recognized by public institutions. A Programme for Regional Reactivation (PRC), has been implemented by the Provincial Council of Biscay, with the aim of promoting self-employment, mainly among young people, long-term unemployed people and those over 45 years old with special difficulties in re-entering the labor market. The purpose of this article is to analyze the PRC as a best practice in social innovation strategy oriented to employment.

Keywords: Employment; Self-employment; Social innovation; Regional development; Public policies; Empowerment.

Sumario. 1. Introducción. 2. El fomento del emprendimiento como buena práctica de innovación social. 3. Metodología. 4. Análisis de caso: Programa de Reactivación Comarcal. 5. Conclusiones. 6. Referencias bibliográficas.

Cómo citar: Azanza Martínez de Luco, G.; Iglesias Sánchez, A.; Henry Moreno, G.; Alejos Garmendia, B. (2020) Desarrollo regional e innovación social en el autoempleo: estudio del caso de los programas de reactivación comarcal como iniciativa pública. REVESCO. Revista de Estudios Cooperativos, vol. 134, e69170. https://dx.doi.org/10.5209/REVE.69170.

\section{Introducción}

En la última década, la crisis financiera ha sacudido fuertemente el mercado laboral, colocando la necesidad de la innovación social en el ámbito de las políticas de empleo en una posición prioritaria. El empleo contribuye al rendimiento económico, a la calidad de vida y a la inclusión social, por lo que es un elemento clave del desarrollo socioeconómico y del bienestar (Eurostat, 2016). En este contexto, la Estrategia Europa 2020 (Comisión Europea, 2010) recoge en sus objetivos y actividades la promoción de una economía sostenible e inclusiva que genere altos niveles de empleo, productividad y cohesión social. La innovación social puede facilitar estos objetivos a través del desarrollo y la implementación de nuevas ideas y soluciones que responden a las necesidades sociales actuales (Comisión Europea, 2013a).

1 Este trabajo está basado en los resultados del proyecto de investigación (Grant Agreement 612870) Social Innovation: Driving Force of Social Change (SI-DRIVE), financiado por la Comisión Europea en el VII Programa Marco. www.si-drive.eu.

2 Universidad de Deusto, España

Dirección de correo electrónico: garazi.azanza@ deusto.es.

Basque Culinary Center, España

Dirección de correo electrónico: aiglesiass@bculinary.com

$4 \quad$ Universidad de Deusto, España

Dirección de correo electrónico: garbine.henry@deusto.es.

5 Agencia Foral de Empleo y Emprendimiento del Departamento de Empleo, Inclusión Social e Igualdad de la Diputación Foral de Bizkaia, España Dirección de correo electrónico: beatriz.a@dema.eus. 
Dichas soluciones deben responder a las características de mercado laboral, que ha evolucionado en los últimos años, reflejando necesidades distintas en el proceso de recuperación económica. En este sentido, el informe Employment Outlook 2018 de la OCDE (2018), refleja un optimismo generalizado entre los países miembros por la recuperación de los niveles de empleo. Así, los mercados laborales se encuentran en niveles anteriores a la crisis económica en cuanto a cantidad de empleo (OCDE, 2018). Sin embargo, las personas jóvenes de 15 a 29 años, las mujeres, especialmente las de 55 a 64 años, y las personas mayores en general son algunos de los grupos más desfavorecidos en el mercado laboral, mostrando aún tasas de empleo bajas (Eurostat, 2016).

En términos generales, el informe de la OCDE (2018) recoge que, si bien el impacto de la crisis financiera en la calidad del empleo y la inclusión persiste, las tasas de empleo son altas en la mayoría de países, y la tasa de desempleo ha vuelto a niveles anteriores a la crisis, situándose aproximadamente 2 puntos porcentuales por encima. También se prevé que las tasas de empleo y desempleo sigan mejorando en 2019. Sin embargo, este optimismo generalizado tiene sus matices. El mismo informe recoge una preocupación con respecto a la calidad y la inclusión en el empleo recuperado. La inseguridad laboral aún no ha alcanzado los niveles pre-crisis y se observa un incremento en la pobreza de la población activa. Además, se observa un empeoramiento significativo de los ingresos de las personas trabajadoras a tiempo parcial en relación con las trabajadoras a tiempo completo, y un incremento del empleo a tiempo parcial involuntario en varios países. El informe también refleja la precaución de esta generalización atendiendo a la situación no tan favorecedora para algunos países europeos como España o Grecia en términos de desempleo y la infrautilización de la población activa (OCDE, 2018).

Como se observa, el crecimiento del empleo no se replica por igual en todos los países miembros. La situación del mercado laboral en España ha mejorado continuamente en los últimos tres años, pero todavía está por debajo de su nivel anterior a la crisis. Además, a la alta tasa de desempleo se suma la alta incidencia de contratos a tiempo parcial y la inseguridad del mercado laboral, el Segundo más bajo en la OCDE después de Grecia (OCDE, 2018). En 2019, aún se espera que España registre una de las tasas de desempleo más altas de la Unión Europea (Comisión Europea, 2018).

Con respecto a los segmentos más afectados por el desempleo, el Anuario Estadístico de España (INE, 2018) refleja una brecha entre las personas mayores y menores de 25 años con respecto al acceso al empleo. Así, la tasa de desempleo de las personas entre 20 y 25 años es del 35,22\%, mientras que para aquellas de 25 a 55 años se sitúa en el 15,92\%. Además, aunque ha habido un descenso considerable en los últimos años, los últimos datos del INE (2018) indican que el desempleo de larga duración se situó en 2017 en un 8,8\% en el caso de las mujeres y en un 6,7\% en el caso de los hombres. Asimismo, las mujeres y los hombres mayores de 50 años presentan los porcentajes más elevados.

En la Comunidad Autónoma de Euskadi (en adelante CAE), donde se sitúa el programa de reactivación objeto de estudio, se respira cierto optimismo en relación con el empleo, siendo la segunda comunidad con la tasa de desempleo más baja, por detrás de la Comunidad Foral de Navarra (INE, 2018). Así, según los datos del segundo trimestre de 2018, la tasa de paro se sitúa en un 10,1\% (EUSTAT, 2018). Tal y como recoge el Informe Laboral Euskadi relativo al primer trimestre 2018 (De La rica, Gorjón y Vega, 2018), las tasas de paro de hombres y mujeres se igualaron en el 15\%. Asimismo, las tasas de paro de los jóvenes se situaron en niveles anteriores a la crisis, aunque todavía están por encima de los colectivos de mayor edad. Con respecto al desempleo de larga duración, pese a haber descendido en los últimos años, la proporción sobre la población parada total aún se sitúa por encima del $60 \%$.

En este contexto, se pone de manifiesto la necesidad de reforzar las políticas activas de empleo para seguir avanzando en la recuperación del mercado laboral a nivel regional, estatal y europeo. Uno de los principales objetivos de la Estrategia Europa 2020 (Comisión Europea, 2013b) es la creación de empleo y, para ello, desarrolla sus orientaciones y programas en materia de empleo, como el Programa para el Empleo y la Innovación Social (EaSi), dirigido a potenciar el crecimiento y la creación de empleo, la movilidad laboral y el progreso social. Para ello, se han creado acciones prácticas tales como las redes de aprendizaje europeas, en las que distintos países miembros comparten buenas prácticas de cómo enfrentar los problemas sociales derivados del desempleo en áreas tan diversas como la gestión de la edad, empoderamiento e inclusión, la problemática del género o emprendimiento inclusivo, entre otras (Oeij y Enciso, 2017). Y, por otra parte, la Comisión Europea ha lanzado políticas específicas para estimular el progreso en estas áreas de trabajo como pueden ser la nueva Agenda Europea para la Integración, Estrategias de Igualdad o la Estrategia de Diversidad Funcional Europea (Oeij y Enciso, 2017).

En España, la Estrategia para la Activación del Empleo 2014-2016 del Servicio Público de Empleo Estatal (SEPE, 2014) recogió los grupos de interés prioritarios para la promoción del empleo y los englobó en a) el desempleo de larga duración, b) las personas desempleadas de baja cualificación, y c) la problemática específica de algunos colectivos que define como personas jóvenes menores de 25 años y personas mayores de 55 años. En esta línea, en el año 2017, las medidas se dirigieron principalmente al empleo de personas jóvenes y al fomento del autoempleo (Eurofound, 2017). 
Más recientemente, la Estrategia Española de Reactivación de Empleo 2017-2020 (RD 1032/2017, de 15 de diciembre) ha hecho hincapié en el refuerzo de los programas orientados a las personas desempleadas de larga duración y mayores de cincuenta años y en personas jóvenes sin cualificación profesional. La concreción anual de la Estrategia se establece en el Plan Anual de Políticas de Empleo (PAPE), confeccionadas anualmente entre el Servicio Público de Empleo Estatal y los servicios públicos de empleo de las comunidades autónomas (RSEE, de 28 de marzo de 2018), cuyos objetivos estratégicos se centran fundamentalmente en los colectivos anteriormente mencionados.

En la CAE, la Estrategia Vasca de Empleo 2020 (EVE 2020) recoge el Plan Estratégico de Empleo 20172020 (Gobierno Vasco, 2018), dirigido a incentivar la creación de empresas y la contratación de personas con menores oportunidades de empleo y ofrecer oportunidades laborales a personas jóvenes. Así, se presenta el fomento del autoempleo como política activa de empleo a través de medidas de apoyo integral al desarrollo de nuevas iniciativas emprendedoras, incluyendo el acompañamiento y asesoramiento en la fase de definición y maduración de la idea y el acceso a la financiación para la puesta en marcha de los proyectos.

En este marco, el presente artículo recoge una iniciativa de carácter regional que pretende fomentar la reactivación económica a través del autoempleo de personas que pertenecen a los segmentos donde el desempleo es más acuciante.

Así, el objeto de este artículo es estudiar la aplicación de la innovación social en políticas de fomento del empleo a través del análisis del Programa de Reactivación Comarcal de Bizkaia (PRC en adelante), recogido como un caso de buena práctica de carácter regional para la promoción del autoempleo y el emprendimiento. El PRC fue puesto en marcha por el Departamento Foral de Empleo, Inclusión Social e Igualdad de la Diputación Foral de Bizkaia ${ }^{6}$ con el objetivo de sensibilizar a las personas desempleadas participantes y formarlas en el manejo de las técnicas y herramientas que permitan crear una empresa, y conseguir que finalicen el programa creando su propio puesto de trabajo, bien como empresa individual, bien como socio o socia de un proyecto empresarial en el que participen varias personas, fomentándose especialmente la creación de cooperativas. Este programa combina la formación grupal, acompañamiento individual y apoyo financiero tanto en la etapa de empoderamiento de la persona como de creación del negocio. En concreto, en el presente artículo se analizan el perfil de las personas participantes, la naturaleza de la motivación hacia el emprendimiento en los proyectos implementados, y el impacto del programa en términos de valoración de las personas participantes y el número y características de proyectos creados.

\section{El fomento del emprendimiento como buena práctica de innovación social}

En los últimos años, la promoción del autoempleo se ha convertido en una de las prioridades de la sociedad debido al reconocimiento de la creación de empresas como motor de crecimiento económico, creación de empleo e innovación (Audretsch, 1995; Kwoka y White, 2001; Carree y Thurik, 2003; Tokila, 2011) y ha ido ganando terreno en el ámbito de las Políticas Activas de Empleo. Sin embargo, también han ido creciendo posiciones críticas con respecto al impacto real del autoempleo en el desarrollo regional, en relación con la falta de viabilidad inicial o pocas expectativas de generación de puestos de trabajo de las empresas de nueva creación o startups (Shane, 2009). De hecho, el último informe del Global Entrepreneurship Monitor (GEM, 2018) recoge que cada vez más personas emprendedoras buscan oportunidades de autoempleo basadas en el propio conocimiento, con bajas expectativas de creación de empleo. Según los últimos datos, en España menos de la mitad de las personas emprendedoras espera generar empleo, y solo un $9 \%$ planea contratar seis o más empleados en los próximos cinco años. Aun así, la realidad es que el desarrollo del emprendimiento ha atraído la atención de académicos, responsables políticos y expertos y la utilización de subsidios para el desarrollo de nuevos negocios está aplicándose de manera muy activa entre los estados de Unión Europea (Molle, 2007; Tokila, 2011).

En este contexto, en el ámbito institucional se observa un interés creciente por las políticas de empleo con enfoque social basadas en la promoción del autoempleo, que pueden tener un papel relevante en el desarrollo regional y la inclusión social (Comisión Europea, 2013a). La economía social y el emprendimiento social proporcionan oportunidades de empleo o servicios para colectivos en riesgo de exclusión, y a menudo están involucradas en iniciativas orientadas al cambio social y a la innovación social (Comisión Europea, 2013a). Así han surgido iniciativas "bottom-up" de innovación social basadas en el emprendimiento para hacer frente a los problemas sociales del mercado de trabajo como pueden ser las empresas sociales que fomentan la integración profesional de personas con diversidad funcional (Davister, Defourny y Gregoire, 2004) o las acciones basadas en el cooperativismo, redes de intercambio sociales, etc. como ejemplos de la utilización

6 Los resultados expuestos en este artículo derivan de un proyecto de investigación realizado en 2016 por la Universidad de Deusto para la Diputación Foral de Bizkaia dentro del marco del Programa Bizkailab. Las autoras quieren agradecer la colaboración de la Diputación Foral de Bizkaia para la realización de este artículo. 
del emprendimiento para resolver problemáticas sociales que el mercado de trabajo por sí mismo, no regula adecuadamente (Castells, 2012).

Las políticas de emprendimiento inclusivo tienen como objetivo garantizar que todas las personas, independientemente de sus características personales y antecedentes, tengan la misma oportunidad de crear su propio negocio (OCDE, 2017a). Los objetivos de este tipo de políticas son mostrar el emprendimiento como una salida laboral y fomentar la motivación hacia el emprendimiento, y abordar las necesidades que afectan en mayor medida a las personas en grupos subrepresentados y desfavorecidos. Esto incluye el acceso a mercados financieros, la adquisición de competencias emprendedoras, la creación de redes y el desarrollo de la cultura emprendedora.

Las políticas de emprendimiento inclusivo en España han experimentado un notable desarrollo en los últimos años. Al mismo tiempo, el aumento del desempleo debido a la crisis económica ha puesto en relieve la necesidad de políticas orientadas a grupos desfavorecidos específicos, en particular a las personas jóvenes, mayores, personas con discapacidad e inmigrantes (OCDE, 2017b).

La naturaleza compleja del emprendimiento plantea considerables desafíos para el diseño de medidas de política económica y requiere de una clara comprensión de los mecanismos y motivaciones que llevan a las personas a emprender. Además, las personas demandantes de empleo que están motivadas a crear su propio negocio deben superar barreras como la falta de competencias emprendedoras y de gestión de empresas, dificultades para acceder a financiación y falta de asesoramiento, entre otras (Comisión Europea, 2014a). Por ello, las políticas de reactivación que ofrecen formación, asesoramiento y ayudas económicas para la puesta en marcha de negocios se presentan como una herramienta valiosa para hacer frente al desempleo.

En esta línea, en el ámbito regional que nos ocupa, Desde la Diputación Foral de Bizkaia, considerando el impacto de este tipo de programas, se ha trabajado en el ámbito de la innovación sociolaboral diseñando y poniendo en marcha distintas iniciativas para apoyar la creación y puesta en marcha de pequeñas empresas y proyectos de autoempleo por personas desempleadas o en riesgo de exclusión, incluyendo asesoramiento y cursos de formación y ayudas a la puesta en marcha y creación de empresas (DF 39/2018, de 20 de marzo). Entre estas iniciativas destaca el PRC objeto de estudio, a raíz del cual actualmente se ha desarrollado el Programa Taldeka-Equipos de emprendimiento (DF 15/2017, de 28 de febrero), que busca la colaboración con instituciones privadas especialistas en alguna de sus distintas dimensiones, reforzando en enfoque sistémico de la innovación social. El público objetivo de estos programas son personas en riesgo de vulnerabilidad, no solo por la edad o la duración de la situación de desempleo, sino también mujeres socialmente vulnerables, profesionales inmigrantes, personas provenientes de puestos de dirección y mandos intermedios en desempleo.

Así pues, el PRC nace con la vocación de dar solución al problema del desempleo y evitar su cronificación, y se recoge como buena práctica de innovación social por su enfoque basado en el acompañamiento, el trabajo a nivel regional, el apoyo a personas en riesgo de vulnerabilidad y la promoción y crecimiento de la economía social y solidaria.

En primer lugar, tal y como se ha señalado anteriormente, una característica del programa es el acompañamiento durante todo el proceso. Según recogen Azanza y Henry (2016) en su revisión de las mejores prácticas europeas, se trataría de un programa innovador que recoge las recomendaciones en este tipo de políticas, al estructurarse en las siguientes fases: 1) Desarrollo de habilidades emprendedoras, 2) Realización del plan de negocio, y 3) Constitución de la empresa y alta de actividad. Así, durante la realización de las fases 1 y 2 las personas participantes reciben una ayuda económica de $350 €$ al mes, y en la fase 3, con la constitución de la empresa o la generación del autoempleo, la persona desempleada recibe una ayuda de 5.000 euros siempre y cuando mantenga la actividad empresarial durante un mínimo de 12 meses.

En segundo lugar, el programa trabaja a nivel regional. Según recoge la Plataforma Social Innovation Exchange en su informe para el desarrollo de la innovación social a nivel regional (Social Innovation Exchange, 2017), dentro de la Unión Europea las regiones tienen un papel esencial para hacer frente a las principales demandas en materia de innovación social como las desigualdades sociales, el envejecimiento de la población y, en el caso que nos ocupa, el desempleo, principalmente, de larga duración. La razón principal es la cercanía a la ciudadanía, que los dota de una mayor capacidad de generar y liderar respuestas innovadoras a estos problemas sociales, y que se ha demostrado la capacidad de las instituciones a nivel regional en materia de empleo y políticas sociales.

En tercer lugar, el PRC objeto de estudio atiende a un público objetivo con mayores problemas de inserción en el mercado de trabajo según las estadísticas europeas y nacionales. Tal y como se menciona anteriormente, a pesar de que Bizkaia tiene uno de los índices más bajos de desempleo en comparación al resto de las provincias españolas (de un 11,63\% en relación al 15,28\% según los datos de INE del segundo trimestre de 2018), según expertos de la propia Diputación, hay un número importante de personas que, por diferentes circunstancias como la escasa cualificación, la edad, el desempleo de larga duración, o la formación en áreas poco demandadas, se van quedando al margen de la recuperación.

Por último, la iniciativa analizada busca la promoción y crecimiento de la economía social y solidaria. El PRC pretende motivar e inducir hacia el emprendimiento y el autoempleo, con especial arreglo a la 
facilitación para la adopción de formas jurídicas del ámbito de la economía social y solidaria, en aras de fomentar un emprendimiento más social y catalizar el surgimiento de iniciativas "bottom up".

\section{Metodología}

Para el estudio de caso se han analizado las ediciones del PRC en el periodo 2012-2015. Así, se ha contado con el total del universo de personas participantes en el PRC en todas sus ediciones hasta 2015, fecha de realización de la encuesta.

La población de estudio la componen las 424 personas participantes (235 hombres, 189 mujeres), que es la totalidad de participantes de los PRC puestos en marcha en diversas comarcas de Bizkaia durante los años 2012, 2013, 2014 y 2015. De los 424 participantes de los PRC objeto de análisis, 202 personas, 112 hombres y 90 mujeres, respondieron a la encuesta. Se trata de una muestra representativa de 202 observaciones con un nivel de confianza del $99 \%$ y un margen de error del 6,6\%.

La información se obtuvo mediante fuentes primarias directas. Se realizó una encuesta telefónica para recoger sus datos sociodemográficos, sus motivaciones y el impacto del PRC.

Se analizaron las siguientes variables:

Datos sociodemográficos: Se recogieron datos sobre la comarca y año de participación en el programa, sexo, edad, nivel de estudios, rama de actividad y situación profesional actual. Dichos datos proporcionan información sobre el perfil de las personas participantes, y su análisis puede orientar estrategias futuras para llegar a los colectivos menos representados.

Motivación para el autoempleo: Se analizó si la motivación para emprender de las personas participantes que crearon su empresa a raíz del programa se debió a una oportunidad de negocio o a una necesidad (por ejemplo, por no tener otra salida laboral). La motivación para el autoempleo puede influir en la supervivencia de los proyectos; la motivación por necesidad se ha relacionado con una menor permanencia y consistencia de las iniciativas emprendedoras (Robichaud et al., 2001). Por ello, se examina la motivación principal de las personas participantes para poder adaptar las acciones formativas y de apoyo.

Impacto del PRC: Se analizó la valoración general del programa en términos de la utilidad percibida y se analizaron el número y las características de los proyectos puestos en marcha a partir de la participación en el programa. Además de recoger datos sobre el número de proyectos puestos en marcha a partir del programa, resulta relevante mostrar la percepción de utilidad por parte de las personas participantes, que pueden haber incrementado su empleabilidad no solo a partir de la creación de proyectos, sino también a nivel formativo, de orientación o de apoyo.

El análisis de datos se llevó a cabo con el paquete estadístico IBM SPSS Statistics y se examinaron estadísticos descriptivos y diferencias de medias (ANOVA de un factor).

\section{Análisis de caso: Programa de Reactivación Comarcal}

\section{Perfil de las personas participantes}

En primer lugar, como parte del análisis del caso, se describe el perfil de las personas participantes en el PRC. Tal y como se ha desarrollado a lo largo del apartado introductorio, la inclusión en el mercado laboral resulta especialmente difícil para algunos colectivos diferenciados. En el caso de la CAE, según recoge el Informe Laboral Euskadi relativo al primer trimestre de 2018 (ISEAK, 2018), se observa una gran incidencia de personas mayores de 44 años, y un aumento en la incidencia de los menores de 25 años entre las personas desempleadas. Del mismo modo, cabe destacar el peso de las personas desempleadas de larga duración es mayor que en otras regiones de España. Todos ellos se consideran colectivos objetivo tal y como recogen las bases del propio PRC.

Dado su impacto en el crecimiento económico, los programas de fomento del autoempleo se consideran una alternativa eficiente a las ayudas al desempleo tradicionales. Este hecho resulta especialmente relevante entre el colectivo objeto del PRC, puesto que, tomando en consideración que la persona que se encuentra en situación de desempleo podría haber puesto en marcha un negocio de todos modos, esto es, sin la participación en el programa (Boone y Van Ours, 2004), diversos estudios demuestran que la duración del emprendimiento, así como el nivel de renta de la persona promotora es mayor cuando forman parte de alguna iniciativa pública de acompañamiento (Tokila, 2011).

En el PRC se observa que las personas participantes responden a perfiles destacados en el ámbito de las políticas de empleo y emprendimiento inclusivo. Así, según recoge la Tabla 1, las principales personas receptoras del programa son personas en desempleo, con un 31,7\% del total y personas que trabajan por cuenta propia con o sin asalariados ascienden al 51\% del total (ver Tabla 1). De las 64 personas en situación de desempleo, la duración media en este estado fue de 23,04 meses (DT = 19,5). 
Tabla. 1. Distribución de la muestra por género, edad, nivel de estudios, sector de actividad y situación profesional (valores porcentuales)

\begin{tabular}{lc}
\hline & Porcentaje \\
\hline Género & \\
Hombre & 55,4 \\
Mujer & 44,6 \\
\hline Edad & \\
Menos de 30 años & 16,3 \\
De 31 a 40 años & 33,7 \\
De 41 a 50 años & 31,7 \\
De 51 a 60 años & 18,3 \\
\hline Nivel de estudios & 32 \\
E. Universitarios & 21 \\
F.P. Grado Superior & 19 \\
F.P. Grado Medio & 13 \\
E. Primarios & \\
\hline Sector de actividad & 31 \\
Comercio, Transporte y Hostelería & 19 \\
Industria, energía y saneamiento & 13 \\
Actividades profesionales y auxiliares & 12 \\
Construcción & 12 \\
Adm. Pública, educación y sanidad & 6 \\
\hline Información y comunicaciones & \\
Situación profesional & 31,4 \\
Trabajo por cuenta propia sin & \\
asalariados/as & 47,5 \\
Trabajo por cuenta propia con & \\
asalariados/as & \\
Desempleo & \\
Trabajo por cuenta ajena eventual & \\
Trabajo por cuenta ajena fijo & \\
Otras situaciones & \\
\hline & \\
\hline
\end{tabular}

Fuente: Elaboración propia.

Según se observa en la Tabla 1, en cuanto a la comparativa entre edad y situación profesional, esta composición no muestra grandes sorpresas salvo, quizás, en el ámbito del desempleo, donde los jóvenes obtienen un menor índice que la media, pero un mayor índice de trabajo eventual, siendo el trabajo asalariado fijo más común en personas entre 51 y 60 años. Por otra parte, se observa un mayor índice de personas en situación de desempleo en el rango de edad entre 41 y 50 años. Por último, las personas que trabajan por cuenta propia responden en su mayoría a edades comprendidas entre 31 y 40 años. Dicha diferencia fue significativa $[\mathrm{F}(3,198)=2,89, \mathrm{p}=0,03]$. No se observaron diferencias significativas entre género y situación profesional $[\mathrm{F}(1,200)=1,55, \mathrm{p}=$ n.s. $]$. 
Tabla. 2. Distribución de la situación profesional por género y edad (valores porcentuales)

\begin{tabular}{lcccccc}
\hline & \multicolumn{3}{c}{ Género } & \multicolumn{5}{c}{ Edad } \\
\hline Situación profesional & Hombres & Mujeres & menos 30 & $31-40$ & $41-50$ & $51-60$ \\
\hline $\begin{array}{l}\text { Trabajo por cuenta propia con } \\
\text { asalariados/as }\end{array}$ & 3,6 & 3,3 & 12,1 & 1,5 & 3,1 & 0,0 \\
Trabajo por cuenta propia sin & 41,1 & 55,6 & 39,4 & 63,2 & 40,6 & 37,8 \\
asalariados/as & 7,1 & 1,1 & 3,0 & 2,9 & 4,7 & 8,1 \\
Trabajo por cuenta ajena fijo & 14,3 & 7,8 & 18,2 & 10,3 & 10,9 & 8,1 \\
Trabajo por cuenta ajena eventual & 31,3 & 32,2 & 27,3 & 22,1 & 40,6 & 37,8 \\
Desempleo & 2,7 & 0 & 0 & 0 & 0 & 8,1 \\
Otras situaciones & & & & & & \\
\hline
\end{tabular}

Fuente: Elaboración propia.

\section{Naturaleza de la motivación hacia el emprendimiento en los proyectos implementados}

En segundo lugar, se analiza la naturaleza de la motivación hacia el emprendimiento en los proyectos implementados. Aunque el desempleo en sí mismo puede considerarse un factor motivador para el emprendimiento, haciendo que el espíritu emprendedor latente se active y lleve a las personas al autoempleo (Thurik, Carree, Stel y Audretsch, 2008), los resultados de otras investigaciones anteriores apuntan a que puede que esta motivación por necesidad no sea la más adecuada para fomentar emprendimientos permanentes y consistentes (Robichaud et al., 2001). En nuestro contexto, el último informe GEM de la CAE (Saiz et al., 2017) recoge que la percepción de la oportunidad como motivador para emprender, remonta desde 2013, a pesar de verse reducida de 2015 a 2016.

En este sentido, tal y como recoge la Tabla 3, parece que en el caso del PRC la motivación de emprender por necesidad se mantiene con diferencia como la principal con respecto a la oportunidad, siendo el 75,2\% de las personas emprendedoras en fase inicial en 2016 motivadas por dicha oportunidad. Por otra parte, atendiendo a rangos de edad, se aprecia una motivación por necesidad ligeramente creciente y proporcional a la edad de la muestra, siendo las personas menores de 30 años las que más emprenden por oportunidad y las mayores de 50 las que más lo hacen por necesidad. Sin embargo, dichas diferencias no fueron significativas $[\mathrm{F}(3,198)=, 69, \mathrm{p}=\mathrm{n} . \mathrm{s}]$. Tampoco se hallaron diferencias significativas entre género y motivación $[\mathrm{F}(1$, 200) $=, 31, \mathrm{p}=$ n.s. $]$.

Tabla 3. Distribución de la motivación para emprender por género y edad (valores porcentuales)

\begin{tabular}{lcccccc}
\hline \multicolumn{4}{c}{ Género } & \multicolumn{5}{c}{ Edad } \\
\hline Motivación & Hombre & Mujer & $\begin{array}{c}\text { Menos de 30 } \\
\text { años }\end{array}$ & De 31 a 40 & De 41 a 50 & De 51 a 60 \\
\hline Oportunidad & 23,2 & 26,7 & 30,3 & 26,5 & 25 & 16,2 \\
Necesidad & 76,8 & 73,3 & 69,7 & 73,5 & 75 & 83,8 \\
\hline
\end{tabular}

Fuente: Elaboración propia.

Impacto del PRC: Valoración general del programa y proyectos creados

En tercer lugar, se analiza el impacto del programa analizando la utilidad percibida por parte de las personas participantes y los proyectos puestos en marcha a partir del PRC. Las personas emprendedoras necesitan una amplia gama de habilidades para poner en marcha su negocio (Unger, Rauch, Frese y Rosenbusch, 2011). Estas habilidades incluyen competencias emprendedoras y de gestión de empresas (Comisión Europea, 2014b). En comparación con otras personas emprendedoras, las personas desempleadas que emprenden por necesidad presentan una mayor necesidad de dichas habilidades (Iyigun y Owen, 1998). Además, las personas desempleadas se enfrentan a dificultades y falta de recursos para empezar sus negocios (Dunn y Holtz-Eakin, 2000; Taylor, 1996). Estos recursos pueden ser tanto financieros como humanos y sociales (Baker y Nelson, 2005; Grichnik et al., 2014). Además, cuanto mayor la duración de la situación de desempleo, mayor es la pérdida de su capital humano por el hecho de no estar activos en el mercado laboral (Ljungqvist y Sargent, 1998).

Por ello, son necesarios los programas que también forman en competencias emprendedoras (Henry et al., 2016), como es el caso de PRC, para proveer de estas herramientas a las personas participantes y prolongar la supervivencia y éxito del emprendimiento. 
Con respecto a la percepción de utilidad del PRC para la creación de autoempleo, de las personas que actualmente están autoempleadas (51\% de las personas encuestadas), el $90 \%$ afirma que ha sido gracias a este Programa objeto de estudio. Este porcentaje o "tasa emprendedora" es mayor que el que se contempla en el conjunto de la población activa en general, donde según últimos datos del GEM (Saiz et al., 2017), en la CAE la tasa de emprendimiento es de 3,3\%. Es decir, la participación en este tipo de proyectos representa un acelerador de la actividad emprendedora.

Realizando un análisis posterior del impacto del programa, de las 202 personas encuestadas, 103 trabajan por cuenta ajena actualmente. En este sentido, se hallaron diferencias significativas con respecto al género $[F(1,171)=4,71, p=0,03]$., la edad $[F(3,168)=3,23, p=0,02]$. y el nivel de estudios $[F(4,168)=5,23, p=$ $0,00]$, siendo la mayoría de las personas emprendedoras mujeres $(54,6 \%)$, personas de 31 a 40 años $(43,29 \%)$ y con estudios universitarios $(41,23 \%)$.

Desde el programa, la falta de actividad emprendedora de casi la mitad de los participantes encuestados no se percibe como fracaso, debido a que se considera que las herramientas, la formación y el asesoramiento ofrecido sirvieron para desarrollar las competencias y aumentar las oportunidades de empleo de las personas participantes. De hecho, la OCDE (2017a) recoge que uno de los resultados de las políticas de emprendimiento inclusivo es la mejora del vínculo con el mercado laboral. Al ayudar a las personas a desarrollar competencia y construir redes, estas iniciativas pueden fomentar la empleabilidad de las personas participantes. Por ello, el éxito de las políticas de emprendimiento inclusivo no solo se mide por el número de personas emprendedoras o proyectos creados, sino también en términos de mejora de la empleabilidad de aquellas personas que han recibido acompañamiento, formación y experiencia emprendedora en dichas iniciativas.

Analizando más en profundidad el impacto del programa en la implementación de los proyectos, atendiendo al género, todas las mujeres que han creado su negocio afirman haberlo hecho a partir de su participación en el programa, mientras que el $88 \%$ de los hombres realizan la misma afirmación. No se obtuvieron datos suficientes para poder discernir la supervivencia de las iniciativas, puesto que, longitudinalmente, solo se ha contado con tres años de observación.

Por último, se analizan las empresas y proyectos creados en el ámbito de la economía social y solidaria a partir del PRC, debido a que el PRC pretende motivar e inducir hacia el emprendimiento y el autoempleo, en aras de promover un crecimiento económico más equitativo y justo con los colectivos que más padecen el desempleo y los problemas sociales de derivados del mismo y de la crisis. Asimismo, este programa recoge también la preocupación de la OCDE (2017) por la redistribución de los beneficios de la recuperación el empleo entre dichos colectivos. Es por estos motivos por los que el programa pone un foco especial en la facilitación para la adopción de formas jurídicas del ámbito de la economía social y solidaria, como son Sociedades Laborales o Cooperativas en aras de fomentar el emprendimiento social.

Sin embargo, atendiendo a los resultados del PRC, las empresas y proyectos producto del programa no se ubican en el ámbito de la economía social y solidaria. Se trata de uno de los principales puntos de mejora del programa, ya que ninguna de las empresas creadas responde a estas características. Esto pudo deberse a que la mayoría de las iniciativas fueron unipersonales, y no se fomentó de manera explícita el emprendimiento colectivo. Así, el objetivo de fomentar sociedades laborales o cooperativas y reforzar la economía social y solidaria, queda pendiente para futuras ediciones.

La ausencia de empresas del ámbito de la economía social y solidaria también podría deberse a las barreras y dificultades que experimentan las personas emprendedoras en el ámbito social. La iniciativa a favor del emprendimiento social (Social Business Initiative) puesta en marcha en 2011, recogió tres líneas de acción que facilitarían la creación y desarrollo de proyectos sociales: mejorar el acceso a la financiación, mejorar el entorno jurídico y mejorar la visibilidad de las empresas sociales (Comisión Europea, 2014c).

De hecho, la creación de empresas sociales puede resultar más difícil debido a las competencias necesarias para la puesta en marcha y gestión de un negocio cuya misión social es tan importante como la económica, y por la dificultad de acceso a capital y fondos por la poca visibilidad y falta de comprensión de las empresas sociales y su valor social (OECD, 2013). Ante las dificultades de trabajar con las instituciones financieras tradicionales, las empresas sociales deben buscar nuevas formas de captar fondos, como podrían ser la financiación solidaria, la inversión institucional, la inversión individual, la filantropía de riesgo, instrumentos de cuasicapital y capital social o mercados de capital social (Mendell y Nogales, 2009) y el crowdfunding. Por ello, la formación y orientación específica sobre este tipo de fuentes de financiación podría facilitar la creación de empresas del ámbito social.

Las empresas sociales tienen características específicas que crean necesidades complejas que exigen soluciones adaptadas a las mismas. Por ello, trabajar aspectos relacionados con las barreras anteriormente mencionadas o plantear programas específicos orientados a la creación de empresas del ámbito de la economía social y solidaria podría fomentar la creación de dichas empresas a partir de políticas activas de empleo. En este contexto, para dar respuesta a las mencionadas necesidades, la Diputación Foral de Bizkaia ha puesto en marcha el Programa de Emprendimiento e Innovación Social, que tiene como objetivo la promoción de la economía social innovadora a través del apoyo a la constitución de entidades sociales 
innovadoras que cuenten con recursos propios adecuados a su objeto y con perspectivas de crecimiento (DF 66/2017, de 16 de mayo).

\section{Conclusiones}

En el ámbito del mercado laboral, el autoempleo se ha convertido en una de las prioridades de las Políticas Activas de Empleo, no sólo por su implicación como motor de crecimiento sino por su capacidad para promocionar un emprendimiento social así como su papel en el empoderamiento de los grupos de interés para la innovación social; esto es, personas en riesgo de exclusión o vulnerabilidad, siendo éstas mujeres, personas migrantes, jóvenes o personas paradas de larga duración, entre otros (Oeij y Enciso, 2017).

El PRC se presenta como un programa atractivo para reactivar y auto-emplear a personas que se encuentran más desfavorecidas en el mercado de trabajo actual. Así, el caso analizado se dirige a perfiles destacados del ámbito de la innovación social. Sin embargo, se identifican líneas de mejora para la aplicación futura de este tipo de programas basados en la innovación social.

En primer lugar, en el caso analizado se detecta que, entre las personas que crearon su empresa a partir del programa, que la necesidad relacionada con su situación de desempleo fue la principal motivación. Dada la naturaleza de esta motivación, en este tipo de programas se debería apoyar la automotivación y trabajar la viabilidad de los proyectos, para garantizar la supervivencia de dichos proyectos y la superación de obstáculos que pueden presentarse a lo largo del proceso emprendedor, y no solo en la creación del proyecto. La supervivencia de los proyectos es un elemento clave a la hora de diseñar programas de reactivación. Por ejemplo, en el caso de New Enterprise Allowance, una iniciativa similar puesta en marcha en Reino Unido que proporciona a las personas destinatarias ayudas económicas durante 26 semanas, así como acceso a un crédito para hacer frente a los costes de lanzamiento del nuevo negocio y formación, mentoring y acompañamiento, se observa que los participantes también crean sus proyectos a partir del programa (OCDE, 2014), pero los negocios generados son de una escala menor y tienen menor potencial de crecimiento que la media. (Ecorys, 2013). Para fomentar la supervivencia de los proyectos, en Austria, en el programa Business Start-up Programme (UGP - Unternehmensgründungsprogramm) (OCDE, 2014) realizan un análisis exhaustivo de la viabilidad de la idea y de las condiciones individuales de la persona participante durante la fase de ejecución, y ofrecen apoyo personalizado además del económico durante el proceso y para la puesta en marcha del negocio.

En segundo lugar, dadas las características derivadas del entorno y de la propia situación de desempleo, el acompañamiento debería ser de una mayor profundidad, de manera que se desarrollen competencias no solo para promover el autoempleo durante el transcurso del programa, sino para promover habilidades de emprendimiento para toda la vida y evitar, así, la cronificación del desamparo que sufren estos grupos de interés en el mercado laboral. Por ejemplo, en Holanda el Bijstandverlening aan zelfstandigen (BBZ) pone énfasis en el desarrollo competencial, incrementando la probabilidad de que las personas participantes desarrollen negocios sostenibles en el medio plazo (OCDE, 2016). Uno de los elementos de esta propuesta es la elevada tasa de flexibilidad en la formación y los servicios de apoyo que utilizan ya que solo dos tercios de las personas participantes hacen uso efectivo del total de este abanico de opciones de apoyo.

Por último, del análisis realizado se extrae que el objetivo de fomentar sociedades laborales o cooperativas y reforzar la economía social y solidaria requiere un esfuerzo específico orientado a las particularidades y dificultades del emprendimiento social, tales como la visibilización y la comunicación del valor social de los proyectos, y el fomento del emprendimiento colectivo. Para ello, resulta necesario crear ecosistemas que fomenten el emprendimiento social, diseñando marcos legales que vayan de la mano de iniciativas para facilitar el acceso al mercado, acciones formativas para el desarrollo de competencias, e instrumentos financieros dirigidos al apoyo de la creación de empresas sociales OCDE (2017c). Futuras investigaciones podrían centrarse en las características y aspectos clave para el éxito de dichos ecosistemas, y el encaje de los programas desarrollados con este objetivo a partir del PRC.

\section{Referencias bibliográficas}

Azanza, G. y Henry, G. (2016) Políticas de empleo dirigidas a la juventud: identificación de buenas prácticas europeas para su aplicación regional o local. DEUSTO Social Impact Briefings, 1, pp. 229-250. DOI: 10.18543/dsib-1(2016)pp229-250.pdf.

Audretsch, D. B. (1995) Innovation and Industry Evolution. Cambridge: MIT Press.

Baker, T. \& Nelson, R. E. (2005) Creating something from nothing: Resource construction through entrepreneurial bricolage. Administrative science quarterly, 50(3), 329-366. DOI: 10.2189/asqu.2005.50.3.329.

Boone, J. y Van Ours, J. C. (2004) Effective active labor market policies. Centre for Economic Policy Research (CEPR) Discussion paper, 4707. Disponible en: http://anon-ftp.iza.org/dp1335.pdf. 
Carree, M. A. y Thurik, A. R. (2003) The impact of entrepreneurship on economic growth. En Audretsch, D. B. y Acs, Z. J. (Eds.) Handbook of Entrepreneurship Research. Kluwer Academic Publishers: Boston/Dordrecht.

Castells, M. (2012) Redes de indignación y esperanza. Madrid: Alianza Editorial.

Comisión Europea (2000) Conclusiones de la presidencia. Consejo Europeo de Lisboa, 23 y 24 de marzo de 2000. Disponible en: http://www.europarl.europa.eu/summits/lis1_es.htm.

Comisión Europea (2010) Europe 2020 A strategy for smart, sustainable and inclusive growth, Communication from the commission, Brussels, 3.3.2010 COM (2010) 2020. Disponible en: https://eurlex.europa.eu/LexUriServ/LexUriServ.do?uri=COM:2010:2020:FIN:EN:PDF.

Comisión Europea (2013a) Guide to Social Innovation. Disponible en: https://ec.europa.eu/eip/ageing/library/guidesocial-innovation_en.

Comisión Europea (2013b) Europa 2020: la estrategia europea de crecimiento. Oficina de Publicaciones de la Unión Europea: Luxemburgo. DOI: 10.2775/39991.

Comisión Europea (2014a) Policy Brief on Access to Business Start-up Finance for Inclusive Entrepreneurship. Oficina de Publicaciones de la Unión Europea: Luxemburgo. Disponible en: https://www.oecd.org/cfe/leed/Finacing\%20inclusive\%20entrepreneurship\%20policy\%20brief\%20EN.pdf.

Comisión Europea (2014b) Activating jobseekers through entrepreneurship: Start-up incentives in Europe. EEPO Review. DOI: 10.2767/23063.

Comisión Europea (2014c) La iniciativa de emprendimiento social de la Comisión Europea. Disponible en: http://ec.europa.eu/internal_market/publications/docs/sbi-brochure/sbi-brochure-web_es.pdf.

Comisión Europea (2018) Council recommendation on the 2018 National Reform Programme of Spain and delivering a council opinion on the 2018 Stability Programme of Spain. Disponible en: https://ec.europa.eu/info/sites/info/files/2018-european-semester-country-specific-recommendation-commissionrecommendation-spain-en.pdf.

Davister, C., Defourny, J. y Grégoire, O. (2004) Work integration social enterprises in the European Union: an overview of existing models. European Research Network (EMES) Working Papers Series No 04/04. Bruselas. Disponible

en: http://orbi.ulg.ac.be/bitstream/2268/90492/1/Work\%20Integration\%20Social\%20Enterprises\%20in\%20the\%20Euro pean\%20Union_An\%20overview\%20of\%20existing\%20models.pdf.

Decreto Foral de la Diputación Foral de Bizkaia 66/2017, de 16 de mayo, por el que se aprueban las bases reguladoras y la convocatoria para el año 2017 del Programa de Emprendimiento e Innovación Social. Boletín Oficial de Bizkaia, 95.

Decreto Foral de la Diputación Foral de Bizkaia 15/2017 de 28 de febrero de 2017, por el que se establecen las bases reguladoras de la concesión de subvenciones para el fomento del autoempleo y la creación de empresas por personas desempleadas y en riesgo de exclusión. Boletín Oficial de Bizkaia, 44,.

Decreto Foral de la Diputación Foral de Bizkaia 39/2018, de 20 de marzo, por el que se establecen las bases reguladoras de la concesión de subvenciones para el fomento del autoempleo y la creación de empresas por personas desempleadas y en riesgo de exclusión, del ejercicio 2018. Boletín Oficial de Bizkaia, 59.

De la Rica, S., Gorjón, L. y Vega, A. (2018) Informe Laboral de Euskadi. Disponible en: http://www.eapmaster.org/informelaboraleuskadi/pdfsinformes/Analisis_PaisVasco_2018_1trim.pdf.

Diario Oficial de la Unión Europea (2012) Versión consolidada del tratado de funcionamiento de la Unión Europea. Diario Oficial de la Unión Europea, C 326/49.

Dunn, T., y Holtz-Eakin, D. (2000) Financial capital, human capital, and the transition to self-employment: Evidence from intergenerational links. Journal of Labor Economics, Vol. 18, $\mathrm{N}^{\mathrm{o}}$ 2, pp. 285-305. DOI: https://doi.org/10.1086/209959.

Ecorys (2013) New Enterprise Allowance: Qualitative evaluation. Research Report No. 836. Department for Work and Pensions. Disponible

en: https://www.gov.uk/government/uploads/system/uploads/attachment_data/file/207518/rrep836.pdf.

Eurofound (2017) Spain: Developments in working life 2017. Disponible en: https://www.eurofound.europa.eu/sites/default/files/wpef18045.pdf.

Eurostat (2016). Smarter, greener, more inclusive? Indicators to support the Europe 2020 Strategy. Disponible en: https://ec.europa.eu/eurostat/documents/3217494/7566774/KS-EZ-16-001-EN-N.pdf/ac04885c-cfff-4f9c-9f30c9337ba929aa.

EUSTAT. (2018) Base de datos en línea. Disponible en: http://eustat.eus.

GEM - Global Entrepreneurship Monitor (2018) Global Report 2017-18. Global Entrepreneurship Research Association. Disponible en: https://www.gemconsortium.org/report/50012.

Gobierno Vasco (2018) Plan Estratégico de Empleo 2017-2020. Disponible en: http://www.euskadi.eus/contenidos/informacion/6199/es_2284/adjuntos/Plan\%20Empleo_2017-2020.pdf.

Grichnik, D., Brinckmann, J., Singh, L. y Manigart, S. (2014) Beyond environmental scarcity: Human and social capital as driving forces of bootstrapping activities. Journal of Business Venturing, Vol. 29, No 2, pp. 310-326. DOI: 10.1016/j.jbusvent.2013.02.006.

Henry, G., Azanza, G. e Iglesias, A. (2016) Evaluación del programa de Reactivación Comarcal en Bizkaia. Proyecto Bizkailab. Bilbao: Universidad de Deusto. 
INE Anuario Estadístico de España 2018. Disponible en: http://www.ine.es/prodyser/pubweb/anuario18/anu18_completo.pdf.

INE (2018) Base de datos en línea. Disponible en: http://www.ine.es/

ISEAK (2018) Informe Laboral Euskadi Segundo trimestre de 2018. Disponible en: http://www.eapmaster.org/informelaboraleuskadi/ultimo.html.

Iyigun, M. F. y Owen, A. L. (1998) Risk, entrepreneurship, and human-capital accumulation. American Economic Review, pp. 454-457. DOI: https://doi.org/10.2139/ssrn.53661.

Kwoka, J. E. y White, L. J. (2001) The new industrial organization and small business. Small Business Economics, Vol. 16, No 1, pp. 21-30. DOI: 10.1023/A:1011172523262.

Ljungqvist, L. y Sargent, T. J. (1998) The European unemployment dilemma. Journal of political Economy, Vol. 106, No 3, pp. 514-550. DOI: 10.1086/250020.

Mendell, M. \& Nogales, R. (2009) Social Enterprises in OECD Member Countries: What are the financial streams? The Changing Boundaries of Social Enterprises, OECD, Paris.

Molle, W. (2007) European cohesion policy. Routledge: New York.

OCDE (2013) Policy Brief on Social https://www.oecd.org/cfe/leed/Social\%20entrepreneurship\%20policy\%20brief\%20EN_FINAL.pdf.

OCDE (2014) The Missing Entrepreneurs: Policies for Inclusive Entrepreneurship in Europe. DOI: http://dx.doi.org/10.1787/9789264213593-en. Disponible en: https://www.oecd.org/industry/the-missingentrepreneurs-2014-9789264213593-en.htm.

OCDE (2016) Inclusive Business Creation: Good Practice Compendium. DOI: http://dx.doi.org/10.1787/9789264251496-en. Disponible en: https://www.oecd.org/economy/inclusive-businesscreation-good-practice-compendium-9789264251496-en.htm.

OCDE (2017a) The missing entrepreneurs 2017. Policies for Inclusive Entrepreneurship. DOI: http://dx.doi.org/10.1787/9789264283602-en. Disponible en: http://www.oecd.org/industry/the-missingentrepreneurs-2017-9789264283602-en.htm.

OCDE (2017b) Inclusive Entrepreneurship Policies, Country Assessment Notes. Spain, 2017. Disponible en: http://www.oecd.org/industry/smes/SPAIN-country-note-2017.pdf.

OCDE (2017c) Boosting Social Enterprise Development. Good practice compendium. DOI: http://dx.doi.org/10.1787/9789264268500-en. Disponible en: https://www.oecd.org/publications/boosting-socialenterprise-development-9789264268500-en.htm.

OCDE (2018) Employment Outlook 2018 Spain. Disponible en: http://www.oecd.org/spain/Employment-OutlookSpain-EN.pdf.

Oeij, P. y Enciso, M. (2017) European Policybrief Social Innovation in Employment. [S.1.]: European Commission. SIDRIVE, Social Innovation: Driving Force of Social Change.

Real Decreto 1032/2017, de 15 de diciembre, por el que se aprueba la Estrategia Española de Activación para el Empleo 2017-2020. Boletín Oficial del Estado 305, pp. 124186-124217.

Resolución de 28 de marzo de 2018, de la Secretaría de Estado de Empleo, por la que se ordena la publicación del Acuerdo de Consejo de Ministros de 27 de marzo de 2018 por el que se aprueba el Plan Anual de Política de Empleo para 2018, según lo establecido en el artículo 11.2 del texto refundido de la Ley de Empleo, aprobado por el Real Decreto Legislativo 3/2015, de 23 de octubre. Boletín Oficial del Estado 77, pp. 34109-34198.

Robichaud, Y., McGraw, E. y Roger, A. (2001) Towards the development of a Measuring Instrument for Entrepreneurial Motivation. Journal of Developmental Entrepreneruship, Agosto 2001, No 6, pp.189-201. DOI: 10.1111/j.1468-0084.1996.mp58002003.x.

Saiz, M, Hoyos, J., González-Pernía, J.L., Peña, I., González, N., Guerrero, M. y Urbano, D. (2017) Global Entrepreneurship Monitor. Comunidad Autónoma del País Vasco. Informe Ejecutivo 2016. Bilbao, España: Publicaciones de la Universidad de Deusto.

SEPE (2014) Estrategia Española de Activación para el Empleo 2014-2016. NIPO: 274-14-191-4. Disponible en: https://www.sepe.es/contenidos/que_es_el_sepe/publicaciones/pdf/pdf_empleo/estrategia_14_16.pdf.

Shane, S. (2009) Why encouraging more people to become entrepreneurs is bad public policy. Small business economics, 33(2), 141-149.

Social Innovation Exchange (2017) SIX Global Council: Regions as contributors to more social Europe - vision from the Basque Country. The Delegation of the Basque Country to the EU. Disponible en: http://www.socialinnovationexchange.org/categories/read/regions-as-contributors-to-more-social-europe-ndashvision-from-the-basque-country.

Taylor, M. (1996) Earnings, independence or unemployment: Why become self-employed?. Oxford Bulletin of Economics and Statistics, Vol. 58, pp. 253-265.

Thurik A. R., Carree, M. A., Van Stel, A. y Audretsch, D. B. (2008) Does self-employment reduce unemployment? Journal of Business Venturing, Vol. 23, No 6, pp. 673-686. DOI: 10.1016/j.jbusvent.2008.01.007.

Tokila, A. (2011) Econometric Studies on Public Support to Entrepreneurship. Tesis doctoral. Universidad de Jyväskylä: Finlandia.

Unger, J. M., Rauch, A., Frese, M. y Rosenbuch, N. (2011) Human capital and entrepreneurial success: A metaanalytical review. Journal of Business Venturing, Vol. 26, No 3, pp. 341-358. DOI: 10.1016/j.jbusvent.2009.09.004. 